\title{
Fitting and Analyzing Randomly Censored Geometric Extreme Exponential Distribution
}

\author{
M. Y. Danish \\ Department of Statistics, Allama Iqbal Open University, Islamabad, Pakistan \\ yameendanish42sb@yahoo.com \\ Muhammad Aslam \\ Department of Statistics, Riphah International University, Islamabad, Pakistan \\ aslamsdqu@yahoo.com
}

\begin{abstract}
The paper presents the Bayesian analysis of two-parameter geometric extreme exponential distribution with randomly censored data. The continuous conjugate prior of the scale and shape parameters of the model does not exist while computing the Bayes estimates, it is assumed that the scale and shape parameters have independent gamma priors. It is seen that the closed-form expressions for the Bayes estimators are not possible; we suggest the Lindley's approximation to obtain the Bayes estimates. However, the Bayesian credible intervals cannot be constructed while using this method, we propose Gibbs sampling to obtain the Bayes estimates and also to construct the Bayesian credible intervals. Monte Carlo simulation study is carried out to observe the behavior of the Bayes estimators and also to compare with the maximum likelihood estimators. One real data analysis is performed for illustration.
\end{abstract}

Keywords: Log-concave density function, Lindley's approximation, Gibbs sampling, Metropolis-Hastings's algorithm, Markov chain Monte Carlo.

\section{Introduction}

There are several censoring mechanisms that are used in survival time studies to reduce the experimental time and cost. The most popular among these are the right censoring schemes because of their crucial importance in reliability and life testing experiments. An observation is said to be right censored at time $t$ if the exact value of it is not known except that it is greater than or equal to $t$. Under type I censoring, a sample of $n$ units is followed until a fixed time $t$. The lifetime of a sampling unit will be known only if it is less than or equal to the predetermined maximum follow-up time $t$. Clinical data is often collected by fixing a maximum follow-up time $t$ for each unit in the study. Under type II censoring, a sample of $n$ units is followed until a fixed number of units $r \leq n$ have experienced the event of interest. In this scheme the number of units experiencing the event is prefixed but the total duration of the study is random. Type II censoring scheme is often used in life testing applications and toxicology experiments. The unified feature of Type I and Type II censoring schemes is that the exact observation window is known for each unit in the sample.

The third type of right censoring is random censoring in which censoring time is not fixed but depends on other random factors which are modeled by independent random variable known as censoring time variable. Consider an example where the patients with colorectal cancer enter simultaneously into the study after their tumors have been removed by surgery and we want to observe their lifetimes but censoring occurs in one of 
the following forms: loss to follow-up (e.g. the patient may decide to move elsewhere), drop out (e.g. due to bad side effects or refusal to participate), death from other diseases or termination of the study. Clearly, these random factors are beyond the control of an investigator and are modeled by a censoring time variable.

In the statistical analysis of failure time data, an investigator is frequently faced with choosing an underlying parametric distribution. In some situations physical considerations determine the distribution to be employed. However, many statistical problems arise from processes which cannot be theoretically linked to a particular parametric distribution. The lack of the general theory for the choice of an underlying parametric distribution has resulted in the usage of distributions which are not necessarily robust to possible alternative distributions. The exponential distribution is commonly used in life testing studies because of its simplicity and its inherent relation to the well defined theory of Poisson processes. It is somewhat surprising to observe that a limited attention has been paid in analyzing randomly censored lifetimes when the lifetimes are not exponential. The reason may be that the analysis becomes too difficult and may not be tractable. However, the density functions of two-parameter lifetime distributions like Weibull, Burr type XII and generalized exponential, geometric extreme exponential, etc., can take different curve shapes and can incorporate increasing, decreasing or constant failure rates as compared to exponential distribution which can incorporate only a constant failure rate.

In this paper, we consider a two-parameter geometric extreme exponential (GEE) distribution introduced by Marshal and Olkin (1997) and further studied extensively by Adamidis et al. (2005). Like Weibull and gamma distributions, GEE distribution can have increasing, constant or decreasing hazard function depending on the shape parameter. For additional properties of GEE distribution, see Marshal and Olkin (1997). Marshal et al. (2001) studied the discrimination between geometric extreme exponential distribution and Weibull, gamma and log-normal distributions as alternatives. Kitidamrongsuk and Siripanich (2010) provided a method for selecting the member of a collection of distributions that best fit a set of observations. They considered the geometric extreme exponential and gamma distributions in their study and proposed a test statistic based on empirical Laplace transform. Pakyari (2010) compared the generalized exponential with geometric extreme exponential and Weibull distributions based on the likelihood ratio and minimum Kolmogorov distance criteria. For the Bayesian analysis of randomly censored generalized exponential and Weibull distributions, readers are referred to Danish and Aslam $(2013,2014)$.

The remainder of the paper is organized as follows. In Section 2, we derive the model and its assumptions. The maximum likelihood (ML) estimation for the unknown parameters is presented in Section 3. Section 4 contains the prior distributions, loss functions, Bayes estimates based on Gibbs sampling and Lindley's approximation. A simulation study is carried out in Section 5. A real data analysis is performed in Section 6 and finally, we conclude the paper in Section 7. 


\section{The model and its assumptions}

Let $X_{1}, X_{2}, \ldots, X_{n}$ be independent and identically distributed random variables with distribution function $F(x)$ and density function $f(x)$. Let $T_{1}, T_{2}, \ldots, T_{n}$ be also independent and identically distributed random variables with distribution function $G(t)$ and density function $g(t)$. In the context of reliability and life testing experiments, $X_{i}$ 's are the true survival times of $n$ individuals censored by $T_{i}$ 's from the right so that one observes only independent and identically distributed random pairs $\left(Y_{1}, D_{1}\right), \ldots,\left(Y_{n}, D_{n}\right)$, where $Y_{i}=$ min $\left(X_{i}, T_{i}\right)$ and $D_{i}=\mathrm{I}\left(X_{i} \leq T_{i}\right)$ is indicator of noncensored observation, for $i=1,2, \ldots, n$. Now it is simple to show that the joint density function of $Y$ and $D$ is

$$
f_{Y, D}(y, d)=\left\{f_{X}(y)\left(1-G_{T}(y)\right)\right\}^{d}\left\{g_{T}(y)\left(1-F_{X}(y)\right)\right\}^{1-d} ; y \geq 0, d=0,1 .
$$

This is the usual model of random censorship studied under nonparametric context by several authors including Kaplan and Meier (1958), Efron (1967), Breslow and Crowley (1974) and Meier (1975). In some situations the censoring time variable $T$ is informative to the survival time variable $X$ through its distribution function. Koziol and Green (1976) introduced a special model under the assumption that $X$ and $T$ are independent and they are connected by the relation

$$
1-G_{T}(y)=\left\{1-F_{X}(y)\right\}^{\beta} \text {. }
$$

for some positive constant $\beta$. The assumption (2) otherwise indicates that the instantaneous event rate is proportional to the instantaneous rate of censoring. Under the assumption (2), the joint density function (1) reduces to

$$
f_{Y, D}(y, d)=f_{X}(y)\left\{1-F_{X}(y)\right\}^{\beta} \beta^{1-d} ; \quad y>0, d=0,1 .
$$

In this paper, we assume that the random variable $X$ follows a two-parameter GEE distribution with shape parameter $\theta$ and scale parameter $\lambda$. The probability density function and cumulative distribution function of GEE distribution are

$$
f_{X}(x ; \theta, \lambda)=\frac{\theta \lambda e^{-\lambda x}}{\left(1-e^{-\lambda x}+\theta e^{-\lambda x}\right)^{2}} ; x>0, \theta, \lambda>0
$$

and

$$
F_{X}(x ; \theta, \lambda)=1-\frac{\theta e^{-\lambda x}}{1-e^{-\lambda x}+\theta e^{-\lambda x}} .
$$

respectively. Using (4) and (5) in expression (3), we have

$$
f_{Y, D}(y, d ; \theta, \lambda, \beta)=\frac{\lambda \theta^{\beta+1} e^{-\lambda(\beta+1) y} \beta^{1-d}}{\left(1-e^{-\lambda x}+\theta e^{-\lambda x}\right)^{(\beta+2)}} ; y>0, d=0,1 .
$$




\section{Maximum likelihood estimation}

In this section, we derive the ML estimators $\hat{\theta}, \hat{\lambda}$ and $\hat{\beta}$ of unknown parameters $\theta, \lambda$ and $\beta$ assuming the model defined in (6) holds. For an observed sample $\left(y_{1}, d_{1}\right), \ldots,\left(y_{n}, d_{n}\right)=(y, d)$ of size $n$ from (6), the likelihood function is

$$
l(\theta, \lambda, \beta) \mid(y, d)=\lambda^{n} \theta^{n(\beta+1)} e^{-\lambda(\beta+1) \sum_{i=1}^{n} y_{i}} \beta^{n-\sum_{i=1}^{n} d_{i}} \prod_{i=1}^{n}\left(1-\bar{\theta} e^{-\lambda y_{i}}\right)^{-(\beta+2)},
$$

where $\bar{\theta}=(1-\theta)$. The log-likelihood function can be written from (7) as

$$
\begin{aligned}
L(\theta, \lambda, \beta) \mid(y, d)=n \ln \lambda+n(\beta+1) \ln \theta & +\left(n-\sum_{i=1}^{n} d_{i}\right) \ln \beta-\lambda(\beta+1) \sum_{i=1}^{n} y_{i} \\
& -(\beta+2) \sum_{i=1}^{n} \ln \left(1-\bar{\theta} e^{-\lambda y_{i}}\right) .
\end{aligned}
$$

Differentiating (8) with respect to $\theta, \lambda, \beta$ and equating the resulting expressions to zero, we have the likelihood equations as

$$
\begin{aligned}
& \frac{n(\beta+1)}{\theta}-(\beta+2) \sum_{i=1}^{n} \frac{e^{-\lambda y_{i}}}{1-\bar{\theta} e^{-\lambda y_{i}}}=0, \\
& \frac{n}{\lambda}-(\beta+1) \sum_{i=1}^{n} y_{i}-(\beta+2) \bar{\theta} \sum_{i=1}^{n} \frac{y_{i} e^{-\lambda y_{i}}}{1-\bar{\theta} e^{-\lambda y_{i}}}=0
\end{aligned}
$$

and

$$
n \ln \theta-\lambda \sum_{i=1}^{n} y_{i}+\frac{n-\sum_{i=1}^{n} d_{i}}{\beta}-\sum_{i=1}^{n} \ln \left(1-\bar{\theta} e^{-\lambda y_{i}}\right)=0 .
$$

All the likelihood equations are nonlinear, so the ML estimates do not exist in closedforms. We suggest the NLP procedure in SAS to compute the ML estimates of the parameters.

\section{Bayesian estimation}

This section deals with loss functions, prior distributions for the unknown parameters and the Bayes estimates using Gibbs sampling procedure and the Lindley's approximation.

\subsection{Loss functions}

In order to select a best decision in decision theory, an appropriate loss function must be specified. The squared error loss function (SELF) is generally used for this purpose. The use of SELF is well justified when over estimation and under estimation of equal magnitude has the same consequences. When the true loss is not symmetric with respect to over estimation and under estimation, asymmetric loss functions are used to represent the consequences of different errors. Nevertheless, we consider both the symmetric and asymmetric loss functions in our Bayesian analysis. 
The SELF is defined as

$$
l_{1}\left(\hat{\theta}_{S E}, \theta\right)=\left(\hat{\theta}_{S E}-\theta\right)^{2}
$$

where $\hat{\theta}_{S E}$ is a decision to estimate parameter $\theta$ under SE loss function. The Bayes estimator of $\theta$ under SELF is

$$
\hat{\theta}_{S E}=E(\theta)
$$

where $E$ denotes the expectation with respect to the posterior distribution of $\theta$.

The second loss function to be considered is the asymmetric precautionary loss function (APLF) defined by

$$
l_{2}\left(\hat{\theta}_{A P}, \theta\right)=\frac{\left(\hat{\theta}_{A P}-\theta\right)^{2}}{\hat{\theta}_{A P}} .
$$

This loss function is a special case of the general class of precautionary loss functions introduced by Norstrom (1996). The Bayes estimator of $\theta$ under APLF is

$$
\hat{\theta}_{A P}=\left[E\left(\theta^{2}\right)\right]^{\frac{1}{2}} \text {. }
$$

Another very useful asymmetric loss is the linear exponential loss function (LELF) introduced by Varian (1975). The LELF with parameters $k_{1}$ and $c$ is defined as

$$
l_{3}(\Delta)=k_{1}\left(e^{c \Delta}-c \Delta-1\right) \quad ; c \neq 0,
$$

where $\Delta=\hat{\theta}_{L E}-\theta$. The sign of parameter $c$ represents the direction of asymmetry and its magnitude reflects the degree of asymmetry. For $c<0$, the underestimation is more serious than the overestimation, and for $c>0$, the overestimation is more serious than the underestimation. For $c$ close to zero, the LE loss function is approximately the squared error loss function. The Bayes estimate of $\theta$ under LELF is

$$
\hat{\theta}_{L E}=-\frac{1}{c} \ln E\left(e^{-c \theta}\right) \text {. }
$$

Still another suitable asymmetric loss function is the general entropy loss function (GELF) proposed by Calabria and Pulcini (1996). The GELF with parameters $q$ and $k_{2}$ is defined as

$$
l_{4}\left(\hat{\theta}_{G E}, \theta\right)=k_{2}\left[\left(\frac{\hat{\theta}_{G E}}{\theta}\right)^{q}-q \ln \left(\frac{\hat{\theta}_{G E}}{\theta}\right)-1\right] ; q \neq 0 .
$$


For $q>0$, a positive error has more serious effect than a negative error and for $q<0$, a negative error has more serious effect than a positive error. The Bayes estimate under GELF is

$$
\hat{\theta}_{G E}=\left[E\left(\theta^{-q}\right)\right]^{-\frac{1}{q}}
$$

\subsection{Prior and posterior distributions}

For the Bayesian estimation of unknown parameters, one needs prior distributions for these parameters. The prior distributions depend upon the knowledge about the parameters and the experience of similar phenomena. However, the specification and formulation of a prior distribution is a difficult job as correctly pointed out by Arnold and Press (1983) that there is no clear cut way in which one can say that one prior is better than the other. The model under consideration has shape and scale parameters; and the continuous conjugate prior for these parameters does not exist. We assume the following independent gamma priors for $\theta, \lambda$ and $\beta$

$$
\left.\begin{array}{ll}
\pi_{1}(\theta)=\frac{b_{1}^{a_{1}}}{\Gamma\left(a_{1}\right)} \theta^{a_{1}-1} e^{-b_{1} \theta} ; & a_{1}, b_{1}, \theta>0 \\
\pi_{2}(\lambda)=\frac{b_{2}^{a_{2}}}{\Gamma\left(a_{2}\right)} \lambda^{a_{2}-1} e^{-b_{2} \lambda} ; & a_{2}, b_{2}, \lambda>0 \\
\pi_{3}(\beta)=\frac{b_{3}^{a_{3}}}{\Gamma\left(a_{3}\right)} \beta^{a_{3}-1} e^{-b_{3} \beta} ; & a_{3}, b_{3}, \beta>0
\end{array}\right\}
$$

The assumptions of independent gamma priors are not unreasonable. Many authors have used the independent gamma priors for the scale and the shape parameters of lifetime distributions, see for example, Berger and Sun (1993), Kundu and Pradhan (2009), Joarder et al. (2011).

The joint prior density of the unknown parameters can be written as

$$
\pi(\theta, \lambda, \beta) \propto \theta^{a_{1}-1} e^{-b_{1} \theta} \lambda^{a_{2}-1} e^{-b_{2} \lambda} \beta^{a_{3}-1} e^{-b_{3} \beta} .
$$

Combining the density function in (7) and the likelihood function in (17), the joint posterior density function of $\theta, \lambda$ and $\beta$ given data is

$$
\begin{aligned}
\pi(\theta, \lambda, \beta \mid y, d) \propto \theta^{a_{1}+n(\beta+1)-1} e^{-b_{1} \theta} e^{-b_{3} \beta} \lambda^{a_{2}+n-1} e^{-\lambda\left(b_{2}+(\beta+1) \sum_{i=1}^{n} y_{i}\right)} \beta^{a_{2}+n-\sum_{i=1}^{n} d_{i}-1} \times & \prod_{i=1}^{n} \frac{1}{\left(1-\bar{\theta} e^{-\lambda y_{i}}\right)^{\beta+2}} .
\end{aligned}
$$

The posterior expectation any function of parameters, say $U(\theta, \lambda, \beta)$, can be written as

$$
\hat{U}_{\beta}(\theta, \lambda, \beta)=E(U(\theta, \lambda, \beta) \mid(y, d))=\frac{\int_{0}^{\infty} \int_{0}^{\infty} \int_{0}^{\infty} U(\theta, \lambda, \beta) \pi(\theta, \lambda, \beta \mid(y, d)) d \theta d \lambda d \beta}{\iint_{0}^{\infty} \int_{0}^{\infty} \int_{0}^{\infty} \pi(\theta, \lambda, \beta \mid(y, d)) d \theta d \lambda d \beta} .
$$


However, it is not possible to evaluate (19) in closed-form. We use two different methods to approximate (19), namely (a) Gibbs sampling and (b) Lindley's approximation.

\subsection{Bayes estimates using Gibbs sampling}

In this section, we obtain the Bayes estimates of $\theta, \lambda$ and $\beta$ under different loss functions using Gibbs sampling procedure. The full conditional forms for $\theta, \lambda$ and $\beta$ up to proportionality can be obtained from (18) as

$$
\begin{aligned}
& \pi_{1}(\theta \mid \lambda, \beta,(y, d)) \propto \theta^{a_{1}+n(\beta+1)-1} e^{-b_{1} \theta} \prod_{i=1}^{n} \frac{1}{\left(1-\bar{\theta} e^{-\lambda y_{i}}\right)^{\beta+2}}, \\
& \left.\pi_{2}(\lambda \mid \theta, \beta,(y, d)) \propto \lambda^{a_{2}+n-1} e^{-\lambda\left(b_{2}+(\beta+1) \sum_{i=1}^{n} y_{i}\right.}\right) \prod_{i=1}^{n} \frac{1}{\left(1-\bar{\theta} e^{-\lambda y_{i}}\right)^{\beta+2}}
\end{aligned}
$$

and

$$
\pi_{3}(\beta \mid \theta, \lambda,(y, d)) \propto \beta^{a_{3}+n-\sum_{i=1}^{n} d_{i}-1} e^{-\beta\left(b_{3}+\lambda \sum_{i=1}^{n} y_{i}-n \ln \theta+\sum_{i=1}^{n} \ln \left(1-\bar{\theta} e^{-\lambda y_{i}}\right)\right)} .
$$

respectively. To obtain the Bayes estimates using Gibbs sampler, it is required to have some mechanism of generating samples from the full conditional distributions. The full conditional form (20) is log-concave if

$$
\sum_{i=1}^{n}\left(\frac{\theta}{e^{\lambda y_{i}}-1+\theta}\right)^{2}<\frac{a_{1}+n(\beta+1)-1}{\beta+2},
$$

which is true for practical situations. Thus the samples of $\theta$ can be generated using the method proposed by Devroye (1984). The full conditional form (21) can be tackled using the Metropolis-Hastings algorithm (see Gilks et al. 1995) with gamma $\left(a_{2}+n, b_{2}+(\beta+1) \sum_{i=1}^{n} y_{i}\right)$ as a candidate-generating density. The procedure is explained in Chib and Greenberg (1995). Finally the full conditional form (22) is gamma density, so the samples of $\beta$ can be easily generated using any of the gamma generating routines. Now following the idea of Geman and Geman (1984) and using (20), (21), (22), it is possible to generate samples of $(\theta, \lambda, \beta)$ from posterior distribution (18) and then to obtain the Bayes estimates and corresponding credible intervals. Starting with suitable choice of initial values, say $\left(\theta_{0}, \lambda_{0}, \beta_{0}\right)$, we suggest the following procedure to generate the posterior samples and then to obtain the Bayes estimates:

1. Generate $\theta_{1}$ from the log-concave density (20) using the method suggested by Devroye (1984).

2. (i) Generate $x$ from gamma $\left(a_{2}+n, b_{2}+\left(\beta_{0}+1\right) \sum_{i=1}^{n} y_{i}\right)$ and $u$ from $\mathrm{U}(0,1)$, 
(ii) If $u<\min (1, d)$ then $\lambda_{1}=x$ else go to (i),

where

$$
d=\frac{\prod_{i=1}^{n}\left(1-\left(1-\theta_{0}\right) e^{-x y_{i}}\right)^{-\beta_{0}-2}}{\prod_{i=1}^{n}\left(1-\left(1-\theta_{0}\right) e^{-\lambda_{0} y_{i}}\right)^{-\beta_{0}-2}} .
$$

3. Generate $\beta_{1}$ from gamma $\left(a_{3}+n-\sum_{i=1}^{n} d_{i}, b_{3}+\lambda \sum_{i=1}^{n} y_{i}-n \ln \theta+\sum_{i=1}^{n} \ln \left(1-\bar{\theta} e^{-\lambda y_{i}}\right)\right)$.

4. Repeat Steps 1-3 M times to obtain $\left(\lambda_{1}, \theta_{1}, \beta_{1}\right), \ldots,\left(\lambda_{M}, \theta_{M}, \beta_{M}\right)$.

5. The Bayes estimates of $\theta, \lambda$ and $\beta$ under SELF can obtained from

$$
\hat{\theta}_{S E}=\frac{1}{M} \sum_{j}^{M} \theta_{j}, \quad \hat{\lambda}_{S E}=\frac{1}{M} \sum_{j}^{M} \lambda_{j}, \quad \hat{\beta}_{S E}=\frac{1}{M} \sum_{j}^{M} \beta_{j} .
$$

6. The Bayes estimates of $\theta, \lambda$ and $\beta$ under APLF function can be obtained from

$$
\hat{\theta}_{A P}=\left(\frac{1}{M} \sum_{j}^{M} \theta_{j}^{2}\right)^{\frac{1}{2}}, \quad \hat{\lambda}_{A P}=\left(\frac{1}{M} \sum_{j}^{M} \lambda_{j}^{2}\right)^{\frac{1}{2}}, \quad \hat{\beta}_{A P}=\left(\frac{1}{M} \sum_{j}^{M} \beta_{j}^{2}\right)^{\frac{1}{2}} .
$$

7. The Bayes estimates of $\theta, \lambda$ and $\beta$ under LELF can be obtained from

$$
\hat{\theta}_{L E}=\frac{-1}{c_{1}} \ln \left(\frac{1}{M} \sum_{j=1}^{M} e^{-c_{1} \theta_{j}}\right), \hat{\lambda}_{L E}=\frac{-1}{c_{2}} \ln \left(\frac{1}{M} \sum_{j=1}^{M} e^{-c_{2} \lambda_{j}}\right), \hat{\beta}_{L E}=\frac{-1}{c_{3}} \ln \left(\frac{1}{M} \sum_{j=1}^{M} e^{-c_{3} \beta_{j}}\right) .
$$

8. The Bayes estimates of $\theta, \lambda$ and $\beta$ under GELF can be obtained from

$$
\hat{\theta}_{G E}=\left[\frac{1}{M} \sum_{j=1}^{M} \theta_{j}^{-q_{1}}\right]^{-\frac{1}{q_{1}}}, \quad \hat{\lambda}_{G E}=\left[\frac{1}{M} \sum_{j=1}^{M} \lambda_{j}^{-q_{2}}\right]^{-\frac{1}{q_{2}}}, \quad \hat{\beta}_{G E}=\left[\frac{1}{M} \sum_{j=1}^{M} \beta_{j}^{-q_{3}}\right]^{-\frac{1}{q_{3}}} .
$$

\subsection{Bayes estimates using Lindley's approximation}

This section provides the Bayes estimates under different loss functions using the Lindley's approximation. Lindley (1980) proposed a procedure to approximate the ratio of two integrals such as (19). The procedure is explained in Appendix.

The Bayes estimates of $\theta, \lambda$ and $\beta$ under SELF using the Lindley's approximation are

$$
\begin{aligned}
& \hat{\theta}_{S E}=\hat{\theta}+\rho_{1} \sigma_{11}+\rho_{2} \sigma_{12}+\rho_{3} \sigma_{13}+\frac{1}{2}\left(A_{1} \sigma_{11}+A_{2} \sigma_{12}+A_{3} \sigma_{13}\right), \\
& \hat{\lambda}_{S E}=\hat{\lambda}+\rho_{1} \sigma_{21}+\rho_{2} \sigma_{22}+\rho_{3} \sigma_{23}+\frac{1}{2}\left(A_{1} \sigma_{21}+A_{2} \sigma_{22}+A_{3} \sigma_{23}\right), \\
& \hat{\beta}_{S E}=\hat{\beta}+\rho_{1} \sigma_{31}+\rho_{2} \sigma_{32}+\rho_{3} \sigma_{33}+\frac{1}{2}\left(A_{1} \sigma_{31}+A_{2} \sigma_{32}+A_{3} \sigma_{33}\right) .
\end{aligned}
$$


The Bayes estimates of $\theta, \lambda$ and $\beta$ under APLF using the Lindley's approximation are

$$
\begin{aligned}
& \hat{\theta}_{B L}=\left[(\hat{\theta})^{2}+2 \hat{\theta}\left(\rho_{1} \sigma_{11}+\rho_{2} \sigma_{12}+\rho_{3} \sigma_{13}\right)+\hat{\theta}\left(A_{1} \sigma_{11}+A_{2} \sigma_{12}+A_{3} \sigma_{13}\right)+\sigma_{11}\right]^{\frac{1}{2}}, \\
& \hat{\lambda}_{B L}=\left[(\hat{\lambda})^{2}+2 \hat{\lambda}\left(\rho_{1} \sigma_{21}+\rho_{2} \sigma_{22}+\rho_{3} \sigma_{23}\right)+\hat{\lambda}\left(A_{1} \sigma_{21}+A_{2} \sigma_{22}+A_{3} \sigma_{23}\right)+\sigma_{22}\right]^{\frac{1}{2}}, \\
& \hat{\beta}_{B L}=\left[(\hat{\beta})^{2}+2 \hat{\beta}\left(\rho_{1} \sigma_{31}+\rho_{2} \sigma_{32}+\rho_{3} \sigma_{33}\right)+\hat{\beta}\left(A_{1} \sigma_{31}+A_{2} \sigma_{32}+A_{3} \sigma_{33}\right)+\sigma_{33}\right]^{\frac{1}{2}} .
\end{aligned}
$$

The Bayes estimates of $\theta, \lambda$ and $\beta$ under LELF using the Lindley's approximation are

$$
\begin{aligned}
& \hat{\theta}_{L E}=\hat{\theta}-\frac{1}{c_{1}} \ln \left[1-c_{1}\left(\rho_{1} \sigma_{11}+\rho_{2} \sigma_{12}+\rho_{3} \sigma_{13}\right)-\frac{c_{1}}{2}\left(A_{1} \sigma_{11}+A_{2} \sigma_{12}+A_{3} \sigma_{13}\right)+\frac{c_{1}^{2} \sigma_{11}}{2}\right], \\
& \hat{\lambda}_{L E}=\hat{\lambda}-\frac{1}{c_{2}} \ln \left[1-c_{2}\left(\rho_{1} \sigma_{21}+\rho_{2} \sigma_{22}+\rho_{3} \sigma_{23}\right)-\frac{c_{2}}{2}\left(A_{1} \sigma_{21}+A_{2} \sigma_{22}+A_{3} \sigma_{23}\right)+\frac{c_{2}^{2} \sigma_{22}}{2}\right], \\
& \hat{\beta}_{L E}=\hat{\beta}-\frac{1}{c_{3}} \ln \left[1-c_{3}\left(\rho_{1} \sigma_{31}+\rho_{2} \sigma_{32}+\rho_{3} \sigma_{33}\right)-\frac{c_{3}}{2}\left(A_{1} \sigma_{31}+A_{2} \sigma_{32}+A_{3} \sigma_{33}\right)+\frac{c_{3}^{2} \sigma_{33}}{2}\right] .
\end{aligned}
$$

The Bayes estimates of $\theta, \lambda$ and $\beta$ under GELF using the Lindley's approximation are

$$
\begin{aligned}
& \hat{\theta}_{G E}=\hat{\theta}\left[1-\frac{q_{1}}{\hat{\theta}}\left(\rho_{1} \sigma_{11}+\rho_{2} \sigma_{12}+\rho_{3} \sigma_{13}\right)-\frac{q_{1}}{2 \hat{\theta}}\left(A_{1} \sigma_{11}+A_{2} \sigma_{12}+A_{3} \sigma_{13}\right)+\frac{q_{1}\left(q_{1}+1\right)}{2 \hat{\theta}^{2}} \sigma_{11}\right]^{\frac{-1}{q_{1}}} \\
& \hat{\lambda}_{G E}=\hat{\lambda}\left[1-\frac{q_{2}}{\hat{\lambda}}\left\{\left(\rho_{1} \sigma_{21}+\rho_{2} \sigma_{22}+\rho_{3} \sigma_{23}\right)+\frac{\left(A_{1} \sigma_{21}+A_{2} \sigma_{22}+A_{3} \sigma_{23}\right)}{2}-\frac{\left(q_{2}+1\right) \sigma_{22}}{2 \hat{\lambda}}\right\}\right]^{\frac{-1}{q_{2}}} \\
& \hat{\beta}_{G E}=\hat{\beta}\left[1-\frac{q_{3}}{\hat{\beta}}\left\{\left(\rho_{1} \sigma_{31}+\rho_{2} \sigma_{32}+\rho_{3} \sigma_{33}\right)-\frac{\left(A_{1} \sigma_{31}+A_{2} \sigma_{32}+A_{3} \sigma_{33}\right)}{2}+\frac{\left(q_{3}+1\right) \sigma_{33}}{2 \hat{\beta}}\right\}\right]^{\frac{-1}{q_{3}}}
\end{aligned}
$$

The closed-form expressions for $A_{1}, A_{2}$ and $A_{3}$ are provided in Appendix.

\section{Simulation}

A simulation study is performed to observe the behavior of the proposed ML estimators and the Bayes estimators based on Gibbs sampling for different sample sizes and for different censoring rates. We consider different sample sizes: $n=20,40$, 60; different censoring rates: $p=0.50,0.80$; different values of loss function parameters: $c=q=-1.5$, $0.9,-0.3,0.3,0.9,1.5$; different combinations of hyperparameters: $a_{1}=0, b_{1}=0, a_{2}=0$, $\mathrm{b}_{2}=0, a_{3}=0, \mathrm{~b}_{3}=0$ (prior-1), $a_{1}=4, \mathrm{~b}_{1}=2, a_{2}=2, \mathrm{~b}_{2}=2, a_{3}=2, \mathrm{~b}_{3}=2$ (prior-2) when $\theta=2, \lambda=1, \beta=1$, prior-1 and prior-2 $\left(a_{1}=3, \mathrm{~b}_{1}=2, a_{2}=2, \mathrm{~b}_{2}=2, a_{3}=1, \mathrm{~b}_{3}=4\right)$ when $\theta=1.5, \lambda=1, \beta=0.25$. Where prior- 1 denotes the noninformative priors for $\theta, \lambda$ and $\beta$ when all the hyperparameters in (17) are zero and prior-2 denotes the informative priors for $\theta, \lambda$ and $\beta$ when the hyperparameters are taken so that the priors' means are the same as the original means. For a particular case, we generate 1000 randomly censored samples from (6) and for each sample we compute the ML estimates, the Bayes estimates 
under different loss functions based on 20,000 MCMC samples with burn-in 10,000 samples. The average ML estimates, average Bayes estimates and the corresponding mean squared errors (MSEs) are obtained for each replicate. The results are reported in Tables 1-3. It is observed that as the sample size increases the biases and the MSEs the estimators decrease. The behavior of Bayes estimators under prior- 1 is very similar to the ML estimators in terms of MSEs. The Bayes estimators under prior- 2 perform quite better than the ML estimators and the Bayes estimators under prior-1. When comparing the Bayes estimators under different loss functions, it is seen that the

Table 1: Average values of the estimators of $\theta$ and the corresponding MSEs (in parenthesis) under (a) prior-1 and (b) prior-2 when $\theta=2$.

(a)

\begin{tabular}{|c|c|c|c|c|c|c|c|c|c|c|}
\hline \multirow[b]{2}{*}{$p$} & \multirow[b]{2}{*}{$n$} & \multirow[b]{2}{*}{ MLE } & \multirow[b]{2}{*}{ SE } & \multirow[b]{2}{*}{ AP } & \multicolumn{3}{|c|}{ LE } & \multicolumn{3}{|c|}{ GE } \\
\hline & & & & & $c=-0.3$ & $\mathrm{c}=0.3$ & $c=0.9$ & $q=-1.5$ & $q=-0.9$ & $q=-0.3$ \\
\hline 0.50 & 20 & $\begin{array}{c}2.965 \\
(3.114)\end{array}$ & $\begin{array}{c}2.879 \\
(3.166)\end{array}$ & $\begin{array}{c}2.579 \\
(1.557)\end{array}$ & $\begin{array}{c}2.478 \\
(1.706)\end{array}$ & $\begin{array}{c}1.763 \\
(0.916)\end{array}$ & $\begin{array}{c}1.336 \\
(0.904)\end{array}$ & $\begin{array}{c}2.341 \\
(1.321)\end{array}$ & $\begin{array}{c}2.024 \\
(1.247)\end{array}$ & $\begin{array}{c}1.870 \\
(1.132)\end{array}$ \\
\hline & 40 & $\begin{array}{c}2.382 \\
(2.388)\end{array}$ & $\begin{array}{c}2.216 \\
(2.281)\end{array}$ & $\begin{array}{c}2.565 \\
(1.601)\end{array}$ & $\begin{array}{c}2.498 \\
(1.736)\end{array}$ & $\begin{array}{c}1.982 \\
(1.001)\end{array}$ & $\begin{array}{c}1.632 \\
(0.796)\end{array}$ & $\begin{array}{c}2.276 \\
(1.219)\end{array}$ & $\begin{array}{c}2.178 \\
(1.160)\end{array}$ & $\begin{array}{c}1.939 \\
(1.142)\end{array}$ \\
\hline & 60 & $\begin{array}{c}2.112 \\
(1.181)\end{array}$ & $\begin{array}{c}2.234 \\
(1.042)\end{array}$ & $\begin{array}{c}2.479 \\
(1.273)\end{array}$ & $\begin{array}{c}2.431 \\
(1.359)\end{array}$ & $\begin{array}{c}2.066 \\
(0.833)\end{array}$ & $\begin{array}{c}1.800 \\
(0.635)\end{array}$ & $\begin{array}{c}2.159 \\
(1.145)\end{array}$ & $\begin{array}{c}2.108 \\
(1.025)\end{array}$ & $\begin{array}{c}2.047 \\
(0.950)\end{array}$ \\
\hline 0.80 & 20 & $\begin{array}{c}2.915 \\
(3.663)\end{array}$ & $\begin{array}{c}2.898 \\
(3.411)\end{array}$ & $\begin{array}{c}2.742 \\
(1.903)\end{array}$ & $\begin{array}{c}2.673 \\
(2.056)\end{array}$ & $\begin{array}{c}1.994 \\
(1.039)\end{array}$ & $\begin{array}{c}1.567 \\
(0.813)\end{array}$ & $\begin{array}{c}2.528 \\
(1.627)\end{array}$ & $\begin{array}{c}2.350 \\
(1.376)\end{array}$ & $\begin{array}{c}1.944 \\
(1.243)\end{array}$ \\
\hline & 40 & $\begin{array}{c}2.528 \\
(2.305)\end{array}$ & $\begin{array}{c}2.339 \\
(2.284)\end{array}$ & $\begin{array}{c}2.624 \\
(1.624)\end{array}$ & $\begin{array}{c}2.582 \\
(1.739)\end{array}$ & $\begin{array}{c}2.136 \\
(0.981)\end{array}$ & $\begin{array}{c}1.830 \\
(0.6897)\end{array}$ & $\begin{array}{c}2.483 \\
(1.439)\end{array}$ & $\begin{array}{c}2.309 \\
(1.257)\end{array}$ & $\begin{array}{c}1.943 \\
(1.055)\end{array}$ \\
\hline & 60 & $\begin{array}{c}2.364 \\
(1.405)\end{array}$ & $\begin{array}{c}2.325 \\
(1.353)\end{array}$ & $\begin{array}{c}2.534 \\
(1.303)\end{array}$ & $\begin{array}{c}2.502 \\
(1.375)\end{array}$ & $\begin{array}{c}2.176 \\
(0.829)\end{array}$ & $\begin{array}{c}1.942 \\
(0.587)\end{array}$ & $\begin{array}{c}2.430 \\
(1.169)\end{array}$ & $\begin{array}{c}2.205 \\
(1.033)\end{array}$ & $\begin{array}{c}2.009 \\
(0.926)\end{array}$ \\
\hline
\end{tabular}

(b)

\begin{tabular}{|c|c|c|c|c|c|c|c|c|c|}
\hline \multirow[b]{2}{*}{$p$} & \multirow[b]{2}{*}{$n$} & \multirow[b]{2}{*}{ SE } & \multirow[b]{2}{*}{ AP } & \multicolumn{3}{|c|}{ LE } & \multicolumn{3}{|c|}{$\mathrm{GE}$} \\
\hline & & & & $\mathrm{c}=-0.3$ & $\mathrm{c}=0.3$ & $\mathrm{c}=0.9$ & $q=-1.5$ & $q=-0.9$ & $q=-0.3$ \\
\hline \multirow[t]{3}{*}{0.50} & 20 & $\begin{array}{c}2.020 \\
(0.047)\end{array}$ & $\begin{array}{c}2.118 \\
(0.106)\end{array}$ & $\begin{array}{c}2.093 \\
(0.103)\end{array}$ & $\begin{array}{c}1.998 \\
(0.082)\end{array}$ & $\begin{array}{c}1.915 \\
(0.079)\end{array}$ & $\begin{array}{c}2.081 \\
(0.096)\end{array}$ & $\begin{array}{c}2.036 \\
(0.089)\end{array}$ & $\begin{array}{c}1.991 \\
(0.085)\end{array}$ \\
\hline & 40 & $\begin{array}{c}2.015 \\
(0.036)\end{array}$ & $\begin{array}{c}2.108 \\
(0.092)\end{array}$ & $\begin{array}{c}2.089 \\
(0.089)\end{array}$ & $\begin{array}{c}1.984 \\
(0.070)\end{array}$ & $\begin{array}{c}1.893 \\
(0.072)\end{array}$ & $\begin{array}{c}2.077 \\
(0.082)\end{array}$ & $\begin{array}{c}2.027 \\
(0.075)\end{array}$ & $\begin{array}{c}1.976 \\
(0.073)\end{array}$ \\
\hline & 60 & $\begin{array}{c}2.004 \\
(0.026)\end{array}$ & $\begin{array}{c}2.105 \\
(0.062)\end{array}$ & $\begin{array}{c}2.082 \\
(0.058)\end{array}$ & $\begin{array}{c}1.964 \\
(0.045)\end{array}$ & $\begin{array}{c}1.863 \\
(0.036)\end{array}$ & $\begin{array}{c}2.068 \\
(0.053)\end{array}$ & $\begin{array}{c}2.011 \\
(0.047)\end{array}$ & $\begin{array}{c}1.954 \\
(0.048)\end{array}$ \\
\hline \multirow[t]{3}{*}{0.80} & 20 & $\begin{array}{c}2.059 \\
(0.060)\end{array}$ & $\begin{array}{c}2.117 \\
(0.126)\end{array}$ & $\begin{array}{c}2.092 \\
(0.124)\end{array}$ & $\begin{array}{c}2.001 \\
(0.101)\end{array}$ & $\begin{array}{c}1.921 \\
(0.096)\end{array}$ & $\begin{array}{c}2.081 \\
(0.117)\end{array}$ & $\begin{array}{c}2.037 \\
(0.110)\end{array}$ & $\begin{array}{c}1.995 \\
(0.105)\end{array}$ \\
\hline & 40 & $\begin{array}{c}2.041 \\
(0.042)\end{array}$ & $\begin{array}{c}2.122 \\
(0.109)\end{array}$ & $\begin{array}{c}2.089 \\
(0.107)\end{array}$ & $\begin{array}{c}1.990 \\
(0.084)\end{array}$ & $\begin{array}{c}1.901 \\
(0.083)\end{array}$ & $\begin{array}{c}2.081 \\
(0.099)\end{array}$ & $\begin{array}{c}2.032 \\
(0.091)\end{array}$ & $\begin{array}{c}1.983 \\
(0.088)\end{array}$ \\
\hline & 60 & $\begin{array}{c}2.035 \\
(0.030)\end{array}$ & $\begin{array}{c}2.114 \\
(0.075)\end{array}$ & $\begin{array}{c}2.081 \\
(0.071)\end{array}$ & $\begin{array}{c}1.962 \\
(0.057)\end{array}$ & $\begin{array}{c}1.862 \\
(0.067)\end{array}$ & $\begin{array}{c}2.066 \\
(0.065)\end{array}$ & $\begin{array}{c}2.009 \\
(0.059)\end{array}$ & $\begin{array}{c}1.953 \\
(0.060)\end{array}$ \\
\hline
\end{tabular}


Bayes estimators under LELF perform better than the rest. The Bayes estimates under LELF and GELF are computed for various values of loss function parameters $c$ and $q$ in the range -1.5 to 1.5 and reported only for which the estimates are appropriate. It is seen that the range of suitable values of loss function parameters is different for the estimation of shape parameter $\theta$, scale parameter $\lambda$ and censoring parameter $\beta$. Since the ML estimator of $\beta$ exists in closed-form, it outperform in all cases. The scale parameter $\lambda$ can be estimated quite accurately under LE loss function $(c=0.3)$ or under GE loss function $(q=-0.9)$ even for sample size as small as 20 . However, no general rule can be drawn for the estimation of shape parameter $\theta$.

Table 2: Average values of the estimators of $\lambda$ and the corresponding MSEs (in parenthesis) under (a) prior-1 and (b) prior-2 when $\lambda=1$.

(a)

\begin{tabular}{|c|c|c|c|c|c|c|c|c|c|c|}
\hline \multirow[b]{2}{*}{$p$} & \multirow[b]{2}{*}{$n$} & \multirow[b]{2}{*}{ MLE } & \multirow[b]{2}{*}{$\mathrm{SE}$} & \multirow[b]{2}{*}{ AP } & \multicolumn{3}{|c|}{$\mathrm{LE}$} & \multicolumn{3}{|c|}{$\mathrm{GE}$} \\
\hline & & & & & $c=-0.9$ & $c=-0.3$ & $c=0.3$ & $q=-1.5$ & $q=-0.9$ & $q=-0.3$ \\
\hline \multirow[t]{3}{*}{0.50} & 20 & $\begin{array}{c}1.247 \\
(0.395)\end{array}$ & $\begin{array}{c}0.915 \\
(0.141)\end{array}$ & $\begin{array}{c}1.019 \\
(0.001)\end{array}$ & $\begin{array}{c}1.032 \\
(0.163)\end{array}$ & $\begin{array}{c}0.954 \\
(0.145)\end{array}$ & $\begin{array}{c}0.878 \\
(0.139)\end{array}$ & $\begin{array}{c}0.984 \\
(0.135)\end{array}$ & $\begin{array}{c}0.900 \\
(0.143)\end{array}$ & $\begin{array}{c}0.799 \\
(0.169)\end{array}$ \\
\hline & 40 & $\begin{array}{c}1.115 \\
(0.145)\end{array}$ & $\begin{array}{c}0.959 \\
(0.114)\end{array}$ & $\begin{array}{c}1.039 \\
(0.109)\end{array}$ & $\begin{array}{c}1.029 \\
(0.123)\end{array}$ & $\begin{array}{c}0.982 \\
(0.116)\end{array}$ & $\begin{array}{c}0.935 \\
(0.113)\end{array}$ & $\begin{array}{c}1.002 \\
(0.109)\end{array}$ & $\begin{array}{c}0.950 \\
(0.115)\end{array}$ & $\begin{array}{c}0.887 \\
(0.130)\end{array}$ \\
\hline & 60 & $\begin{array}{c}1.078 \\
(0.095)\end{array}$ & $\begin{array}{c}0.983 \\
(0.086)\end{array}$ & $\begin{array}{c}1.034 \\
(0.082)\end{array}$ & $\begin{array}{c}1.027 \\
(0.089)\end{array}$ & $\begin{array}{c}0.997 \\
(0.086)\end{array}$ & $\begin{array}{c}0.968 \\
(0.085)\end{array}$ & $\begin{array}{c}1.010 \\
(0.083)\end{array}$ & $\begin{array}{c}0.977 \\
(0.086)\end{array}$ & $\begin{array}{c}0.939 \\
(0.094)\end{array}$ \\
\hline \multirow[t]{3}{*}{0.80} & 20 & $\begin{array}{c}1.187 \\
(0.250)\end{array}$ & $\begin{array}{c}0.958 \\
(0.118)\end{array}$ & $\begin{array}{c}1.043 \\
(0.116)\end{array}$ & $\begin{array}{c}1.034 \\
(0.131)\end{array}$ & $\begin{array}{c}0.984 \\
(0.121)\end{array}$ & $\begin{array}{c}0.933 \\
(0.116)\end{array}$ & $\begin{array}{c}1.003 \\
(0.115)\end{array}$ & $\begin{array}{c}0.958 \\
(0.119)\end{array}$ & $\begin{array}{c}0.882 \\
(0.132)\end{array}$ \\
\hline & 40 & $\begin{array}{c}1.086 \\
(0.096)\end{array}$ & $\begin{array}{c}0.998 \\
(0.077)\end{array}$ & $\begin{array}{c}1.043 \\
(0.076)\end{array}$ & $\begin{array}{c}1.038 \\
(0.082)\end{array}$ & $\begin{array}{c}1.011 \\
(0.078)\end{array}$ & $\begin{array}{c}0.984 \\
(0.076)\end{array}$ & $\begin{array}{c}1.021 \\
(0.076)\end{array}$ & $\begin{array}{c}0.993 \\
(0.078)\end{array}$ & $\begin{array}{c}0.961 \\
(0.082)\end{array}$ \\
\hline & 60 & $\begin{array}{c}1.059 \\
(0.063)\end{array}$ & $\begin{array}{c}1.011 \\
(0.055)\end{array}$ & $\begin{array}{c}1.039 \\
(0.055)\end{array}$ & $\begin{array}{c}1.037 \\
(0.058)\end{array}$ & $\begin{array}{c}1.019 \\
(0.056)\end{array}$ & $\begin{array}{c}1.002 \\
(0.054)\end{array}$ & $\begin{array}{c}1.026 \\
(0.055)\end{array}$ & $\begin{array}{c}1.008 \\
(0.055)\end{array}$ & $\begin{array}{c}0.988 \\
(0.056)\end{array}$ \\
\hline
\end{tabular}

(b)

\begin{tabular}{|c|c|c|c|c|c|c|c|c|c|}
\hline \multirow[b]{2}{*}{$p$} & \multirow[b]{2}{*}{$n$} & \multirow[b]{2}{*}{ SE } & \multirow[b]{2}{*}{ AP } & \multicolumn{3}{|c|}{ LE } & \multicolumn{3}{|c|}{$\mathrm{GE}$} \\
\hline & & & & $\mathrm{c}=-0.3$ & $\mathrm{c}=0.3$ & $\mathrm{c}=0.9$ & $q=-1.5$ & $q=-0.9$ & $q=-0.3$ \\
\hline \multirow[t]{3}{*}{0.50} & 20 & $\begin{array}{c}1.017 \\
(0.023)\end{array}$ & $\begin{array}{c}1.049 \\
(0.262)\end{array}$ & $\begin{array}{c}1.027 \\
(0.024)\end{array}$ & $\begin{array}{c}1.007 \\
(0.021)\end{array}$ & $\begin{array}{c}0.987 \\
(0.020)\end{array}$ & $\begin{array}{c}1.033 \\
(0.024)\end{array}$ & $\begin{array}{c}1.014 \\
(0.022)\end{array}$ & $\begin{array}{c}0.994 \\
(0.021)\end{array}$ \\
\hline & 40 & $\begin{array}{c}1.007 \\
(0.017)\end{array}$ & $\begin{array}{c}1.030 \\
(0.018)\end{array}$ & $\begin{array}{c}1.014 \\
(0.017)\end{array}$ & $\begin{array}{c}0.999 \\
(0.016)\end{array}$ & $\begin{array}{c}0.985 \\
(0.015)\end{array}$ & $\begin{array}{c}1.018 \\
(0.017)\end{array}$ & $\begin{array}{c}1.004 \\
(0.016)\end{array}$ & $\begin{array}{c}0.990 \\
(0.016)\end{array}$ \\
\hline & 60 & $\begin{array}{c}1.003 \\
(0.016)\end{array}$ & $\begin{array}{c}1.022 \\
(0.017)\end{array}$ & $\begin{array}{c}1.009 \\
(0.016)\end{array}$ & $\begin{array}{c}0.997 \\
(0.015)\end{array}$ & $\begin{array}{c}0.986 \\
(0.015)\end{array}$ & $\begin{array}{c}1.013 \\
(0.016)\end{array}$ & $\begin{array}{c}1.001 \\
(0.016)\end{array}$ & $\begin{array}{c}0.989 \\
(0.016)\end{array}$ \\
\hline \multirow[t]{3}{*}{0.80} & 20 & $\begin{array}{c}1.019 \\
(0.028)\end{array}$ & $\begin{array}{c}1.044 \\
(0.031)\end{array}$ & $\begin{array}{c}1.027 \\
(0.029)\end{array}$ & $\begin{array}{c}1.012 \\
(0.027)\end{array}$ & $\begin{array}{c}0.996 \\
(0.025)\end{array}$ & $\begin{array}{c}1.032 \\
(0.030)\end{array}$ & $\begin{array}{c}1.017 \\
(0.028)\end{array}$ & $\begin{array}{c}1.001 \\
(0.027)\end{array}$ \\
\hline & 40 & $\begin{array}{c}1.011 \\
(0.018)\end{array}$ & $\begin{array}{c}1.027 \\
(0.020)\end{array}$ & $\begin{array}{c}1.016 \\
(0.019)\end{array}$ & $\begin{array}{c}1.005 \\
(0.018)\end{array}$ & $\begin{array}{c}0.995 \\
(0.017)\end{array}$ & $\begin{array}{c}1.019 \\
(0.019)\end{array}$ & $\begin{array}{c}1.009 \\
(0.018)\end{array}$ & $\begin{array}{c}0.998 \\
(0.018)\end{array}$ \\
\hline & 60 & $\begin{array}{c}1.008 \\
(0.015)\end{array}$ & $\begin{array}{c}1.021 \\
(0.016)\end{array}$ & $\begin{array}{c}1.0112 \\
(0.015)\end{array}$ & $\begin{array}{c}1.003 \\
(0.015)\end{array}$ & $\begin{array}{c}0.995 \\
(0.015)\end{array}$ & $\begin{array}{c}1.014 \\
(0.015)\end{array}$ & $\begin{array}{c}1.006 \\
(0.015)\end{array}$ & $\begin{array}{c}0.998 \\
(0.015)\end{array}$ \\
\hline
\end{tabular}


Table 3: Average values of the estimators of $\beta$ and the corresponding MSEs (in parenthesis) under (a) prior-1 and (b) prior-2 when $\beta=1, \beta=0.25$.

(a)

\begin{tabular}{|c|c|c|c|c|c|c|c|c|c|c|}
\hline \multirow[b]{2}{*}{$p$} & \multirow[b]{2}{*}{$n$} & \multirow[b]{2}{*}{ MLE } & \multirow[b]{2}{*}{ SE } & \multirow[b]{2}{*}{ AP } & \multicolumn{3}{|c|}{$\mathrm{LE}$} & \multicolumn{3}{|c|}{ GE } \\
\hline & & & & & $c=-0.3$ & $c=0.3$ & $c=0.9$ & $q=-1.5$ & $q=-0.9$ & $q=-0.3$ \\
\hline \multirow[t]{3}{*}{0.50} & 20 & $\begin{array}{c}1.007 \\
(0.001)\end{array}$ & $\begin{array}{c}0.970 \\
(0.001)\end{array}$ & $\begin{array}{c}1.049 \\
(0.003)\end{array}$ & $\begin{array}{c}1.015 \\
(0.003)\end{array}$ & $\begin{array}{c}0.985 \\
(0.001)\end{array}$ & $\begin{array}{c}0.955 \\
(0.002)\end{array}$ & $\begin{array}{c}0.995 \\
(0.003)\end{array}$ & $\begin{array}{c}0.965 \\
(0.005)\end{array}$ & $\begin{array}{c}0.953 \\
(0.006)\end{array}$ \\
\hline & 40 & $\begin{array}{c}1.003 \\
(0.000)\end{array}$ & $\begin{array}{l}1.021 \\
(0.00)\end{array}$ & $\begin{array}{c}1.044 \\
(1.002)\end{array}$ & $\begin{array}{c}1.019 \\
(0.001)\end{array}$ & $\begin{array}{c}0.996 \\
(0.000)\end{array}$ & $\begin{array}{c}0.974 \\
(0.001)\end{array}$ & $\begin{array}{c}1.016 \\
(0.001)\end{array}$ & $\begin{array}{c}1.003 \\
(0.000)\end{array}$ & $\begin{array}{c}0.980 \\
(0.001)\end{array}$ \\
\hline & 60 & $\begin{array}{c}1.002 \\
(0.000)\end{array}$ & $\begin{array}{c}1.007 \\
(0.000)\end{array}$ & $\begin{array}{c}1.019 \\
(0.001)\end{array}$ & $\begin{array}{c}1.012 \\
(0.000)\end{array}$ & $\begin{array}{c}0.995 \\
(0.000)\end{array}$ & $\begin{array}{c}0.979 \\
(0.001)\end{array}$ & $\begin{array}{c}1.012 \\
(0.001)\end{array}$ & $\begin{array}{c}1.001 \\
(0.000)\end{array}$ & $\begin{array}{c}0.983 \\
(0.000)\end{array}$ \\
\hline \multirow[t]{3}{*}{0.80} & 20 & $\begin{array}{c}0.251 \\
(0.000)\end{array}$ & $\begin{array}{c}0.253 \\
(0.000)\end{array}$ & $\begin{array}{c}0.282 \\
(0.003)\end{array}$ & $\begin{array}{c}0.256 \\
(0.003)\end{array}$ & $\begin{array}{c}0.251 \\
(0.001)\end{array}$ & $\begin{array}{c}0.247 \\
(0.002)\end{array}$ & $\begin{array}{c}0.268 \\
(0.001)\end{array}$ & $\begin{array}{c}0.250 \\
(0.000)\end{array}$ & $\begin{array}{c}0.232 \\
(0.003)\end{array}$ \\
\hline & 40 & $\begin{array}{c}0.251 \\
(0.000)\end{array}$ & $\begin{array}{c}0.252 \\
(0.000)\end{array}$ & $\begin{array}{c}0.275) \\
(0.002)\end{array}$ & $\begin{array}{c}0.255 \\
(0.000)\end{array}$ & $\begin{array}{c}0.250 \\
(0.000)\end{array}$ & $\begin{array}{c}0.248 \\
(0.000)\end{array}$ & $\begin{array}{c}0.256 \\
(0.000)\end{array}$ & $\begin{array}{c}0.253 \\
(0.000)\end{array}$ & $\begin{array}{c}0.244 \\
(0.001)\end{array}$ \\
\hline & 60 & $\begin{array}{c}0.250 \\
(0.000)\end{array}$ & $\begin{array}{c}0.251 \\
(0.000)\end{array}$ & $\begin{array}{c}0.264) \\
(0.000)\end{array}$ & $\begin{array}{c}0.253 \\
(0.000)\end{array}$ & $\begin{array}{c}0.251 \\
(0.000)\end{array}$ & $\begin{array}{c}0.249 \\
(0.000)\end{array}$ & $\begin{array}{c}0.252 \\
(0.000)\end{array}$ & $\begin{array}{c}0.249 \\
(0.000)\end{array}$ & $\begin{array}{c}0.241 \\
(0.001)\end{array}$ \\
\hline
\end{tabular}

(b)

\begin{tabular}{|c|c|c|c|c|c|c|c|c|c|}
\hline \multirow[b]{2}{*}{$p$} & \multirow[b]{2}{*}{$n$} & \multirow[b]{2}{*}{ SE } & \multirow[b]{2}{*}{ AP } & \multicolumn{3}{|c|}{$\mathrm{LE}$} & \multicolumn{3}{|c|}{ GE } \\
\hline & & & & $c=-0.3$ & $c=0.3$ & $c=0.9$ & $q=-1.5$ & $q=-0.9$ & $q=-0.3$ \\
\hline \multirow[t]{3}{*}{0.50} & 20 & $\begin{array}{c}1.026 \\
(0.001)\end{array}$ & $\begin{array}{c}1.079 \\
(0.007)\end{array}$ & $\begin{array}{c}1.043 \\
(0.003)\end{array}$ & $\begin{array}{c}1.010 \\
(0.001)\end{array}$ & $\begin{array}{c}0.980 \\
(0.001)\end{array}$ & $\begin{array}{c}1.053 \\
(0.004)\end{array}$ & $\begin{array}{c}1.021 \\
(0.001)\end{array}$ & $\begin{array}{c}0.990 \\
(0.001)\end{array}$ \\
\hline & 40 & $\begin{array}{c}1.022 \\
(0.001)\end{array}$ & $\begin{array}{c}1.057 \\
(0.003)\end{array}$ & $\begin{array}{c}1.033 \\
(0.001)\end{array}$ & $\begin{array}{c}1.011 \\
(0.000)\end{array}$ & $\begin{array}{c}0.991 \\
(0.000)\end{array}$ & $\begin{array}{c}1.039 \\
(0.002)\end{array}$ & $\begin{array}{c}1.018 \\
(0.001)\end{array}$ & $\begin{array}{c}0.997 \\
(0.000)\end{array}$ \\
\hline & 60 & $\begin{array}{c}1.018 \\
(0.000)\end{array}$ & $\begin{array}{c}1.044 \\
(0.002)\end{array}$ & $\begin{array}{c}1.026 \\
(0.001)\end{array}$ & $\begin{array}{c}1.010 \\
(0.000)\end{array}$ & $\begin{array}{c}0.995 \\
(0.000)\end{array}$ & $\begin{array}{c}1.031 \\
(0.001)\end{array}$ & $\begin{array}{c}1.015 \\
(0.000)\end{array}$ & $\begin{array}{c}1.000 \\
(0.000)\end{array}$ \\
\hline \multirow[t]{3}{*}{0.80} & 20 & $\begin{array}{c}0.253 \\
(0.000)\end{array}$ & $\begin{array}{c}0.268 \\
(0.000)\end{array}$ & $\begin{array}{c}0.254 \\
(0.000)\end{array}$ & $\begin{array}{c}0.252 \\
(0.000)\end{array}$ & $\begin{array}{c}0.249 \\
(0.000)\end{array}$ & $\begin{array}{c}0.261 \\
(0.000)\end{array}$ & $\begin{array}{c}0.251 \\
(0.000)\end{array}$ & $\begin{array}{c}0.241 \\
(0.000)\end{array}$ \\
\hline & 40 & $\begin{array}{c}0.252 \\
(0.000)\end{array}$ & $\begin{array}{c}0.257 \\
(0.000)\end{array}$ & $\begin{array}{c}0.253 \\
(0.000)\end{array}$ & $\begin{array}{c}0.250 \\
(0.000)\end{array}$ & $\begin{array}{c}0.248 \\
(0.000)\end{array}$ & $\begin{array}{c}0.259 \\
(0.000)\end{array}$ & $\begin{array}{c}0.251 \\
(0.000)\end{array}$ & $\begin{array}{c}0.244 \\
(0.000)\end{array}$ \\
\hline & 60 & $\begin{array}{c}0.250 \\
(0.000)\end{array}$ & $\begin{array}{c}0.253 \\
(0.000)\end{array}$ & $\begin{array}{c}0.251 \\
(0.000)\end{array}$ & $\begin{array}{c}0.250 \\
(0.000)\end{array}$ & $\begin{array}{c}0.250 \\
(0.000)\end{array}$ & $\begin{array}{c}0.256 \\
(0.000)\end{array}$ & $\begin{array}{c}0.251 \\
(0.000)\end{array}$ & $\begin{array}{c}0.246 \\
(0.000)\end{array}$ \\
\hline
\end{tabular}

\section{Data Analysis}

In this section, we analyze a real data set from Fleming and Harrington (1991). The data belongs to Group IV of the Primary Biliary Cirrhosis (PBC) liver study conducted by Mayo Clinic. The event of interest is time to death of PBC Patients. The data on the survival times (in days) of 36 patients who had the highest category of bilirubin are: 400, $77,859,71,1037,1427,733,334,41,51,549,1170,890,1413,853,216,1882^{+}, 1067^{+}$, 131, 223, 1827, 2540, 1297, 264, 797, 930, 1329+, 264, 1350, 1191, 130, 943, 974, 790, $1765^{+}, 1320^{+}$. The observations with ' + ' indicate censored times. For computational ease, 
each observation is divided by 1000 . Since we do not have any prior information about the unknown parameters, we use the noninformative priors for $\theta, \lambda$ and $\beta$, that is $a_{1}=b_{1}=a_{2}=b_{2}=a_{3}=b_{3}=0$ for Bayes estimates. We compute the ML estimates and the Bayes estimates under SELF, APLF, LELF and GELF using Gibbs sampling procedure and Lindley's approximation. To test the goodness of fit of the model to this data, we compute the Kolomogorov-Smirnov D statistics and the associated p-values. The results are given in Table 4. Based on the Kolomogorov-Smirnov test, we can say that the test is nonsignificance for all the methods. However, the Bayes estimates under LELF based on Gibbs sampling fit the data quite well.

Table 4: The ML estimates and the Bayes estimates, Kolomogorov-Smirnov D statistics and the associated $\boldsymbol{p}$-values based on different methods for real data

\begin{tabular}{lcccccc}
\hline Method & LF & $\theta$ & $\lambda$ & $\beta$ & K-S D & $p$-value \\
\hline MLE & - & 3.9724 & 1.8703 & 0.1666 & 0.1841 & 0.2442 \\
Bayes (MCMC) & SE & 4.1970 & 1.7720 & 0.1724 & 0.2022 & 0.1555 \\
& AP & 5.0912 & 1.8385 & 0.1921 & 0.2203 & 0.0970 \\
& LE & 2.6158 & 1.6604 & 0.1693 & 0.1376 & 0.5936 \\
& GE & 3.6282 & 1.7118 & 0.1587 & 0.1729 & 0.3154 \\
Bayes (Lindley) & SE & 4.2438 & 1.7953 & 0.2519 & 0.1976 & 0.1777 \\
& AP & 3.3703 & 1.6751 & 0.1529 & 0.1891 & 0.2202 \\
& LE & 2.7547 & 1.7146 & 0.2522 & 0.1505 & 0.4806 \\
& GE & 3.4534 & 1.9195 & 0.1930 & 0.1621 & 0.3927 \\
\hline
\end{tabular}

\section{Conclusion}

In this paper we compared the maximum likelihood estimators and the Bayes estimators of geometric extreme exponential distribution parameters under random censorship. For this the squared error, asymmetric precautionary, linear exponential and general entropy loss functions are considered using informative and noninformative priors. It is observed that the Bayes estimates of unknown parameters cannot be obtained in closed-forms, we used Gibbs sampling and Lindey's approximation to obtain the approximate Bayes estimates. To observe the behavior of the estimators based on different methods, a simulation study is performed for different sample sizes, for different censoring rates and for different loss function parameters of linear exponential and general entropy loss functions. It is observed that as the sample size increases the MSEs of the estimators decrease. The ML estimates and the Bayes estimates under noninformative priors on the unknown parameters behave approximately in similar manner. However, the Bayes estimators under independent gamma priors perform quite better than the ML estimators and the Bayes estimators under noninformative priors. It is seen that the scale parameter $\lambda$ and the censoring parameter $\beta$ can be estimated quite effectively under LELF and GELF with appropriate choice of corresponding parameters. This is true for both the informative 
and noninformative priors and for sample sizes as small as 20 . However, we cannot find a general rule for the estimation of shape parameter $\theta$ even for sample sizes as large as 60 . One real data analysis is performed to illustrate various methods. It is seen that all Kolomogorov-Smirnov test of fit is non-significance for all the methods. However, the Bayes estimate under LELF based on Gibbs sampling fit the data quite better than the rest.

\section{Appendix}

The posterior expectation given in (18), using the notations $(\theta, \lambda, \beta)=\left(\theta_{1}, \theta_{2}, \theta_{3}\right)$ and $\rho\left(\theta_{1}, \theta_{2}, \theta_{3}\right)=\ln \pi\left(\theta_{1}, \theta_{2}, \theta_{3}\right)$, can be written as

$$
\hat{U}_{B}\left(\theta_{1}, \theta_{2}, \theta_{3}\right)=\frac{\int_{\left(\theta_{1}, \theta_{2}, \theta_{3}\right)} U\left(\theta_{1}, \theta_{2}, \theta_{3}\right) e^{L\left(\theta_{1}, \theta_{2}, \theta_{3}\right)+\rho\left(\theta_{1}, \theta_{2}, \theta_{3}\right)} d\left(\theta_{1}, \theta_{2}, \theta_{3}\right)}{\int_{\left(\theta_{1}, \theta_{2}, \theta_{3}\right)} e^{L\left(\theta_{1}, \theta_{2}, \theta_{3}\right)+\rho\left(\theta_{1}, \theta_{2}, \theta_{3}\right)} d\left(\theta_{1}, \theta_{2}, \theta_{3}\right)} .
$$

For sufficiently large $\mathrm{n}$, the expression (34) is evaluated by Lindley's approximation as

$$
\begin{aligned}
\hat{U}_{B}\left(\theta_{1}, \theta_{2}, \theta_{3}\right)=U\left(\hat{\theta}_{1}, \hat{\theta}_{2}, \hat{\theta}_{3}\right) & +\left(U_{1} d_{1}+U_{2} d_{2}+U_{3} d_{3}+d_{4}+d_{5}\right)+\frac{1}{2}\left[A_{1}\left(U_{1} \sigma_{11}+U_{2} \sigma_{12}+U_{3} \sigma_{13}\right)\right. \\
& \left.+A_{2}\left(U_{1} \sigma_{21}+U_{2} \sigma_{22}+U_{3} \sigma_{23}\right)+A_{3}\left(U_{1} \sigma_{31}+U_{2} \sigma_{32}+U_{3} \sigma_{33}\right)\right]
\end{aligned}
$$

where $\hat{\theta}_{1}, \hat{\theta}_{2}, \hat{\theta}_{3}$ are the ML estimates of $\theta_{1}, \theta_{2}, \theta_{3}, d_{i}=\rho_{1} \sigma_{i 1}+\rho_{2} \sigma_{i 2}+\rho_{3} \sigma_{i 3}, \quad i=1,2,3$,

$$
\begin{aligned}
& d_{4}=U_{12} \sigma_{12}+U_{13} \sigma_{13}+U_{23} \sigma_{23}, d_{5}=\frac{1}{2}\left(U_{11} \sigma_{11}+U_{22} \sigma_{22}+U_{33} \sigma_{33}\right), \\
& A_{1}=\sigma_{11} L_{111}+2 \sigma_{12} L_{121}+2 \sigma_{13} L_{131}+2 \sigma_{23} L_{231}+\sigma_{22} L_{221}+\sigma_{33} L_{331}, \\
& A_{2}=\sigma_{11} L_{112}+2 \sigma_{12} L_{122}+2 \sigma_{13} L_{132}+2 \sigma_{23} L_{232}+\sigma_{22} L_{222}+\sigma_{33} L_{332}, \\
& A_{3}=\sigma_{11} L_{113}+2 \sigma_{12} L_{123}+2 \sigma_{13} L_{133}+2 \sigma_{23} L_{233}+\sigma_{22} L_{223}+\sigma_{33} L_{333}, \\
& U_{i}=\frac{\partial U\left(\theta_{1}, \theta_{2}, \theta_{3}\right)}{\partial \theta_{i}}, \rho_{i}=\frac{\partial \rho\left(\theta_{1}, \theta_{2}, \theta_{3}\right)}{\partial \theta_{i}}, L_{i j k}=\frac{\partial^{3} L\left(\theta_{1}, \theta_{2}, \theta_{3}\right)}{\partial \theta_{i} \partial \theta_{j} \partial \theta_{k}}, i, j, k=1,2,3 .
\end{aligned}
$$

Moreover $\sigma_{i j}$ is $(\mathrm{i}, \mathrm{j})$ th element of minus the inverse of the Fisher information matrix and all the quantities are evaluated at $\left(\hat{\theta}_{1}, \hat{\theta}_{2}, \hat{\theta}_{3}\right)$.

In our case

$$
\begin{aligned}
\rho_{1}= & \frac{a_{1}-1}{\hat{\theta}}-b_{1}, \quad \rho_{2}=\frac{a_{2}-1}{\hat{\lambda}}-b_{2}, \quad \rho_{3}=\frac{a_{3}-1}{\hat{\beta}}-b_{3}, \\
A_{1}= & \frac{2 n(\beta+1) \sigma_{11}}{\hat{\theta}^{3}}-2(\beta+2) \sum_{i=1}^{n} \frac{\left(\sigma_{11} e^{-\lambda y_{i}}+2 y_{i} \sigma_{12}\right) e^{-2 \lambda y_{i}}}{\left(1-\bar{\theta} e^{-\lambda y_{i}}\right)^{3}} \\
& -\sigma_{22}(\beta+2) \sum_{i=1}^{n} \frac{\left(1+\bar{\theta} e^{-\lambda y_{i}}\right) y_{i}^{2} e^{-\lambda y_{i}}}{\left(1-\bar{\theta} e^{-\lambda y_{i}}\right)^{3}}+2 \sigma_{23} \sum_{i=1}^{n} \frac{y_{i} e^{-\lambda y_{i}}}{\left(1-\bar{\theta} e^{-\lambda y_{i}}\right)^{2}}
\end{aligned}
$$




$$
\begin{aligned}
A_{2}= & \frac{2 n \sigma_{22}}{\hat{\lambda}^{3}}-2 \sigma_{11}(\beta+2) \sum_{i=1}^{n} \frac{y_{i} e^{-2 \lambda y_{i}}}{\left(1-\bar{\theta} e^{-\lambda y_{i}}\right)^{3}}+2 \bar{\theta} \sigma_{23} \sum_{i=1}^{n} \frac{y_{i}^{2} e^{-\lambda y_{i}}}{\left(1-\bar{\theta} e^{-\lambda y_{i}}\right)^{2}} \\
& -(\beta+2) \sum_{i=1}^{n} \frac{\left(2 \sigma_{12}+\bar{\theta} y_{i} \sigma_{22}\right) y_{i}^{2} e^{-\lambda y_{i}}\left(1+\bar{\theta} e^{-\lambda y_{i}}\right)}{\left(1-\bar{\theta} e^{-\lambda y_{i}}\right)^{3}} \\
A_{3}= & \frac{2\left(n-\sum_{i=1}^{n} d_{i}\right) \sigma_{33}}{\hat{\beta}^{3}}-\frac{n \sigma_{11}}{\hat{\theta}^{2}}+\sum_{i=1}^{n} \frac{e^{-\lambda y_{i}}\left(\sigma_{11} e^{-\lambda y_{i}}+2 \sigma_{12} y_{i}+\bar{\theta} y_{i}^{2} \sigma_{22}\right)}{\left(1-\bar{\theta} e^{-\lambda y_{i}}\right)^{2}}
\end{aligned}
$$

\section{References}

1. Adamidis, K., Dimitrakopoulou, T. and Loukas, S. (2005). On an extension of the exponential-geometric distribution. Statistics and Probability Letters, 73, 259-262.

2. Arnold, B. C. and Press, S. J. (1983). Bayesian inference for Pareto populations, J. Econometrics, 21, 287-306.

3. Berger, J. O. and Sun, D. (1993). Bayesian analysis for the poly-Weibull distribution. nal of American Statistical Association, 88, 1412-1418.

4. Breslow, N. and Crowley, J. (1974). A large sample study of the life table and product limit estimates under random censorship, Annals of Statistics, 2, 437-453.

5. Calabria, R. and Pulcini, G. (1996). Point estimation under asymmetric loss functions for left truncated exponential samples. Communication in StatisticsTheory and Methods, 25, 585-600.

6. Chib, S. and Greenberg, E. (1995). Understanding the Metropolis-Hastings algorithm. The American Statistician, 49, 327-335.

7. Danish, M. Y. and Aslam, M. (2013). Bayesian estimation for randomly censored generalized exponential distribution under asymmetric loss functions. Journal of Applied Statistics, 40, 1106-1119.

8. Danish, M. Y. and Aslam, M. (2014). Bayesian inference for the randomly censored Weibull distribution. Journal of Statistical Computation and Simulation, 84, 215-230.

9. Devroye, L. (1984). A simple algorithm for generating random variates with a logconcave density. Computing, 33, 247-257.

10. Effron, B. (1967). The two-sample problem with censored data. Proceeding of the $5^{\text {th }}$ Berkeley Symposium, 4, 831-853.

11. Fleming, T. R. and Harrington, D. P. (1991). Counting processes and survival analysis. New York: Wiley.

12. Geman, S. and Geman, A. (1984). Stochastic relaxation, Gibbs distributions and the Bayesian restoration of images. IEEE Transactions on Pattern Analysis and Machine Intelligence, 6, 721-740.

13. Gilks, W. R., Richardson, S. and Spiegelhalter, D. J. (1995). Markov chain Monte Carlo in Practice. London: Chapman and Hall. 
14. Joarder, A., Krishna, H. and Kundu, D. (2011). Inference on Weibull parameters with conventional type I censoring. Computational Statistics and Data Analysis, 55, $1-11$.

15. Kaplan, E. L. and Meier, P. (1958). Nonparametric estimation from incomplete observations. Journal of American Statistical Association, 53, 457-481.

16. Kitidamrongsuk, P. and Siripanich, P. (2010). Selecting among families of lifetime distributions. In: Proc. $6^{\text {th }}$ IMT-GT Conf. Math. Stat. Applications. Universiti Tunku Abdul Rahman, Kuala Lumpur, Malaysia, 367-376.

17. Kundu, D. and Pradhan, B. (2011). Bayesian analysis of progressively censored competing risks data. Sankhya, 73, 276-296.

18. Lindley, D. V. (1980). Approximate Bayes methods. Trabajos de Estadistica, 31, 223-237.

19. Marshal, A. W. and Olkin, I. (1997). A new method for adding a parameter to a family of distributions with applications to the exponential and Weibull families. Biometrika, 84, 641-652.

20. Marshal, A. W., Meza, J. C. and Olkin, I. (2001). Can data recognize its parent distribution? Journal of Computation and Graphical Statistics, 10, 555-580.

21. Meier, P. (1975). Estimation of a distribution function from incomplete observations. In J. Ghani (Ed.), Perspectives in probability and statistics (pp. 6787). London: Academic Press.

22. Norstrom, J. G. (1996). The use of precautionary loss functions in risk analysis. IEEE Tran. Rel., 45, 400-403.

23. Pakyari, P. (2010). Discriminating between generalized exponential, geometric extreme exponential and Weibull distributions. Journal of Statistical Computation and Simulation, 80, 1403-1412.

24. Varian, H. R. (1975). A Bayesian approach to real state assessment. In S. E. Feinberg, and A. Zellner (Eds.), Studies in Bayesian Econometrics and Statistics (pp. 195-208). North-Holland: Amsterdam. 